\title{
Systematic identification of microplastics in abyssal and hadal sediments of the Kuril Kamchatka trench.
}

Serena M. Abel a, b, c, Sebastian Primpke ${ }^{b}$, Ivo Int-Veen ${ }^{d}$, Angelika Brandt ${ }^{a, c}$, Gunnar Gerdts ${ }^{b}$.

${ }^{6}$ Department of Microbial Ecology, Biologische Anstalt Helgoland, Alfred Wegener Institute, Helmholtz Centre for Polar and Marine Research, Kurpromenade 201,27498, Helgoland,

Germany,

¿Goethe University Frankfurt, Institute for Ecology, Diversity and Evolution, Max-von-Lauest
d Thünen-Institute of Fisheries Ecology, Herwigstraße 31,27572 Bremerhaven, Germany.

Abstract number: 337476

\section{introduction}

The occurrence of microplastics throughout marine environments worldwide, from pelagic to benthic habitats, has become serious cause for concern. Hadal zones were recently described as the "trash bins of the oceans" and ultimate sink for marine plastic debris. The Kuril region covers substantial area of the North Pacific Ocean and is characterised by high biological productivity, intense marine traffic through the Kuril straits, and anthropogenic activity. Strong tidal currents and eddy activity, as well as the influence of Pacific currents, have the potential for long distance transport and retention of microplastics in this area. The Kuril Kamchatka Trench might accumulate microplastics from the surrounding environments and act as the final sink for high quantities of microplastics

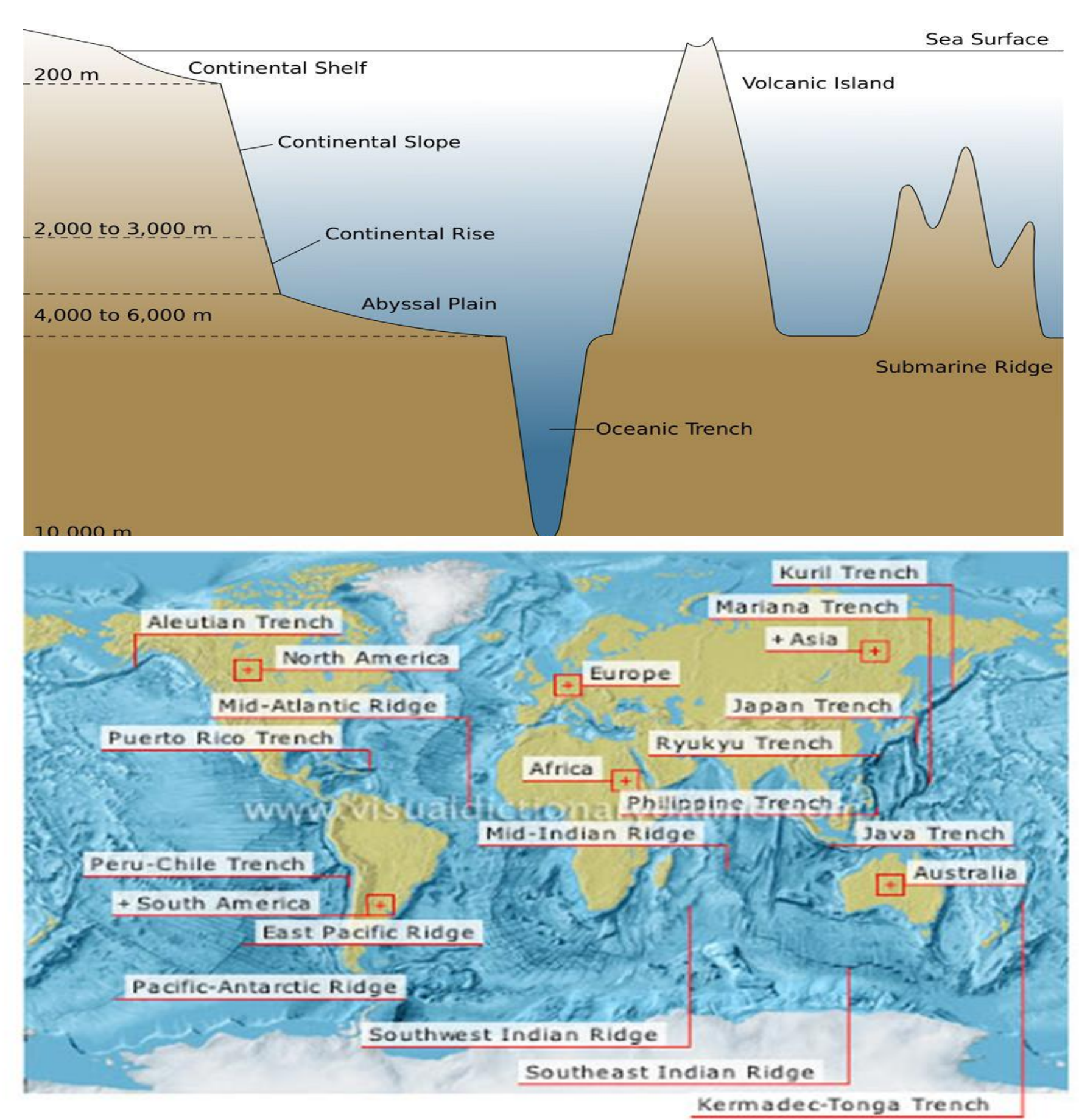

The aim of this study was to quantify the MP present in the deep KKT, to characterise the MP particles by size and polymer type and to contribute to a further understanding of MP accumulation in deep-sea trenches. To examine the potential inaccuracies of aliquot-based estimations, samples were both aliquot sub-sampling and in toto sample analysis were performed on each sample.

\section{Materials and methods}
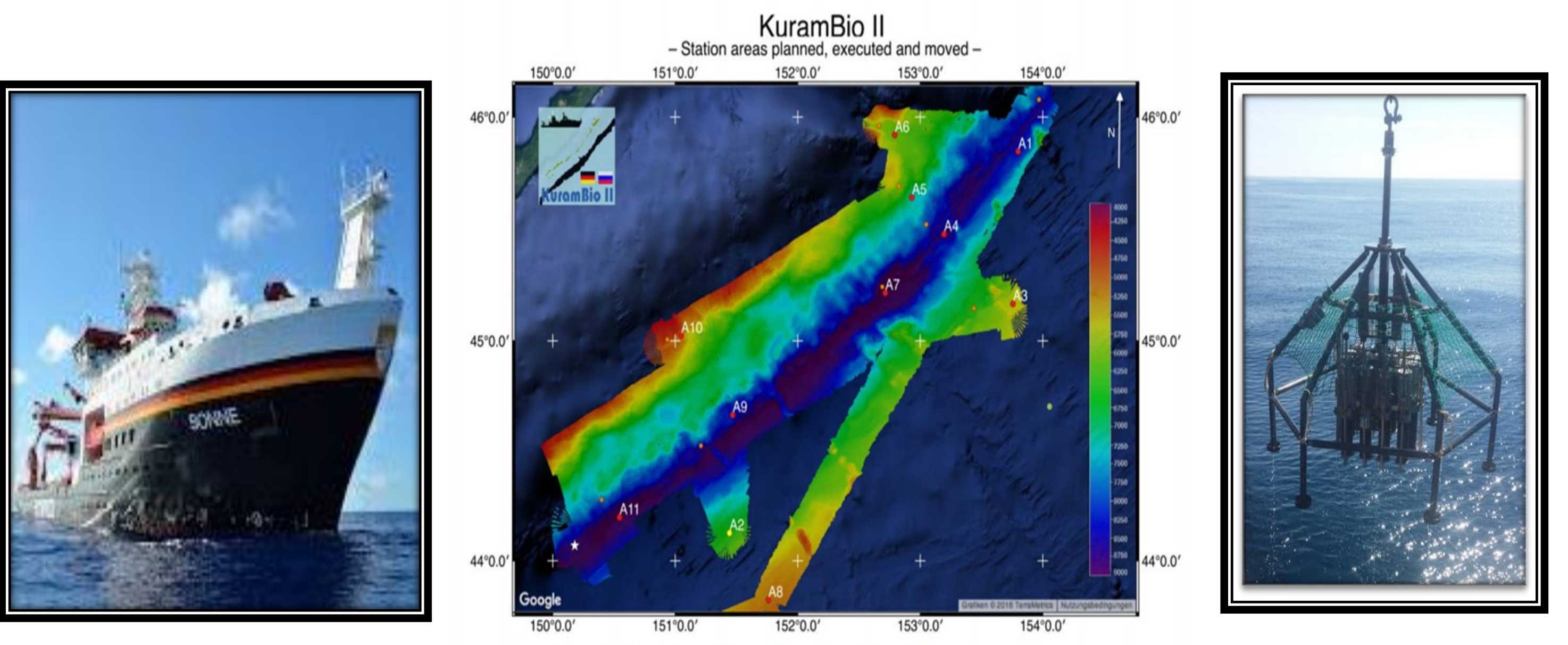

The samples were collected from the KKT (northwest Pacific Ocean) in summer 2016, onboard the RV Sonne, during the deep-sea expedition SO-250 KuramBio Il, using a Multi-corer (MUC, version: 2011-K2 ×100; OKTOPUS GmbH, Kiel, Germany)

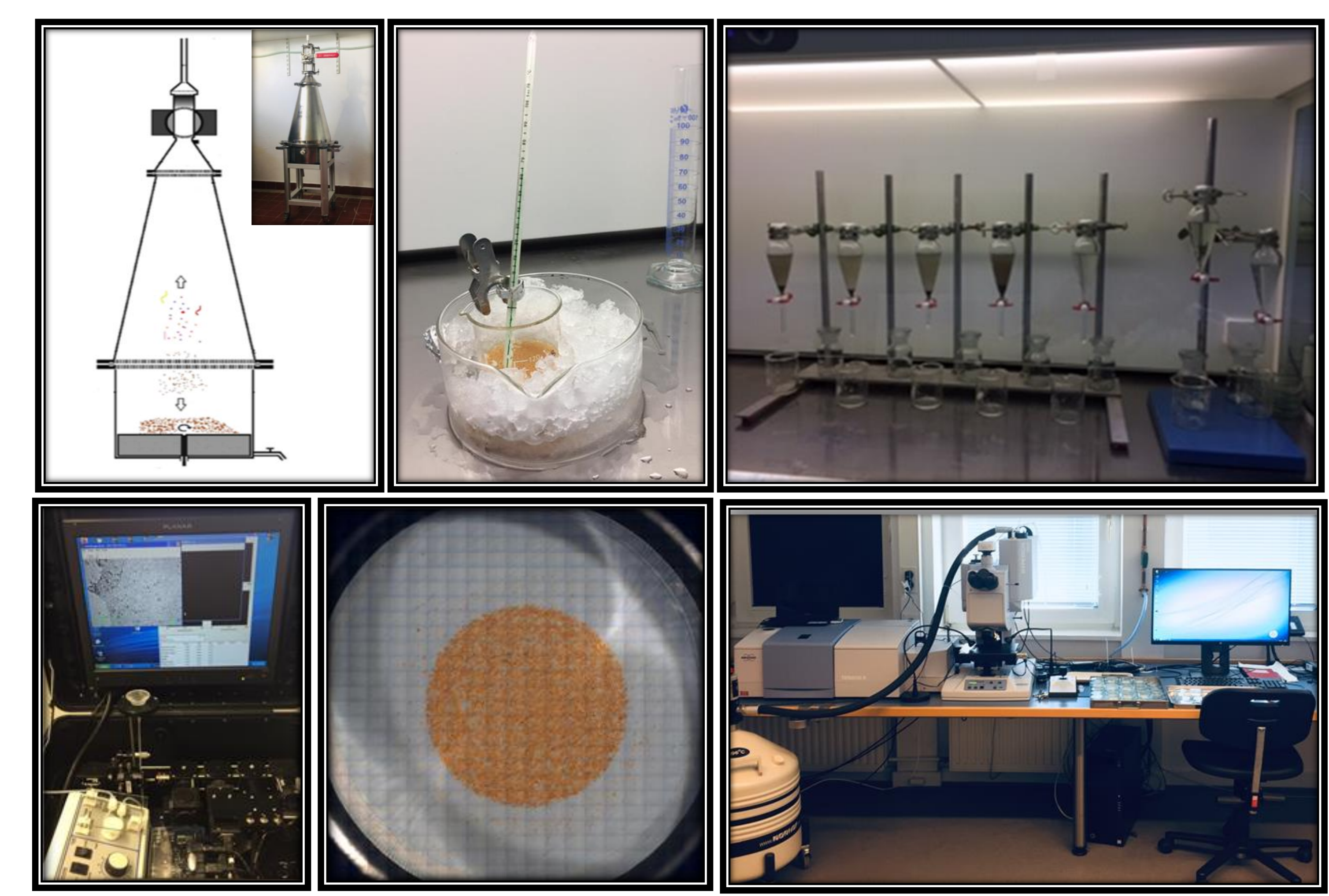

A density separation was then performed on each individual sample using a MPSS device (Micro Plastic Sediment Separator, Hydro-Bios Apparatebau GmbH, Kiell. Fenton's treatment was applied to remove organic material. $A$ A second density-separation step was performed to remove any remaining inorganic material. FlowCam (Fluid Imaging Technologies, Portable version IV, Scarborough, Maine, US) was used to quantify the particle identification,
was applied.

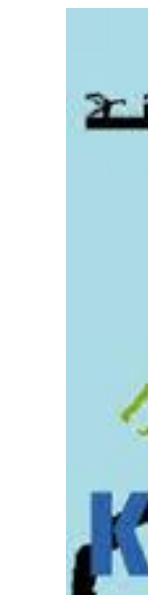
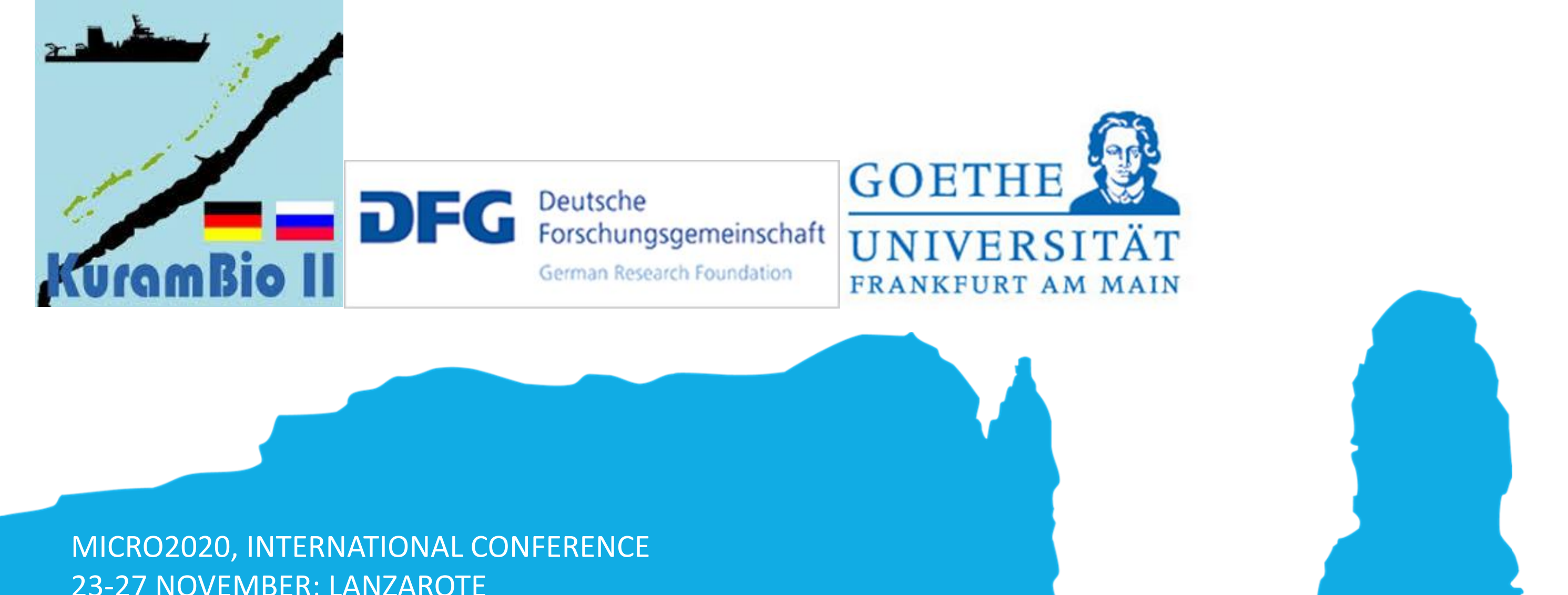

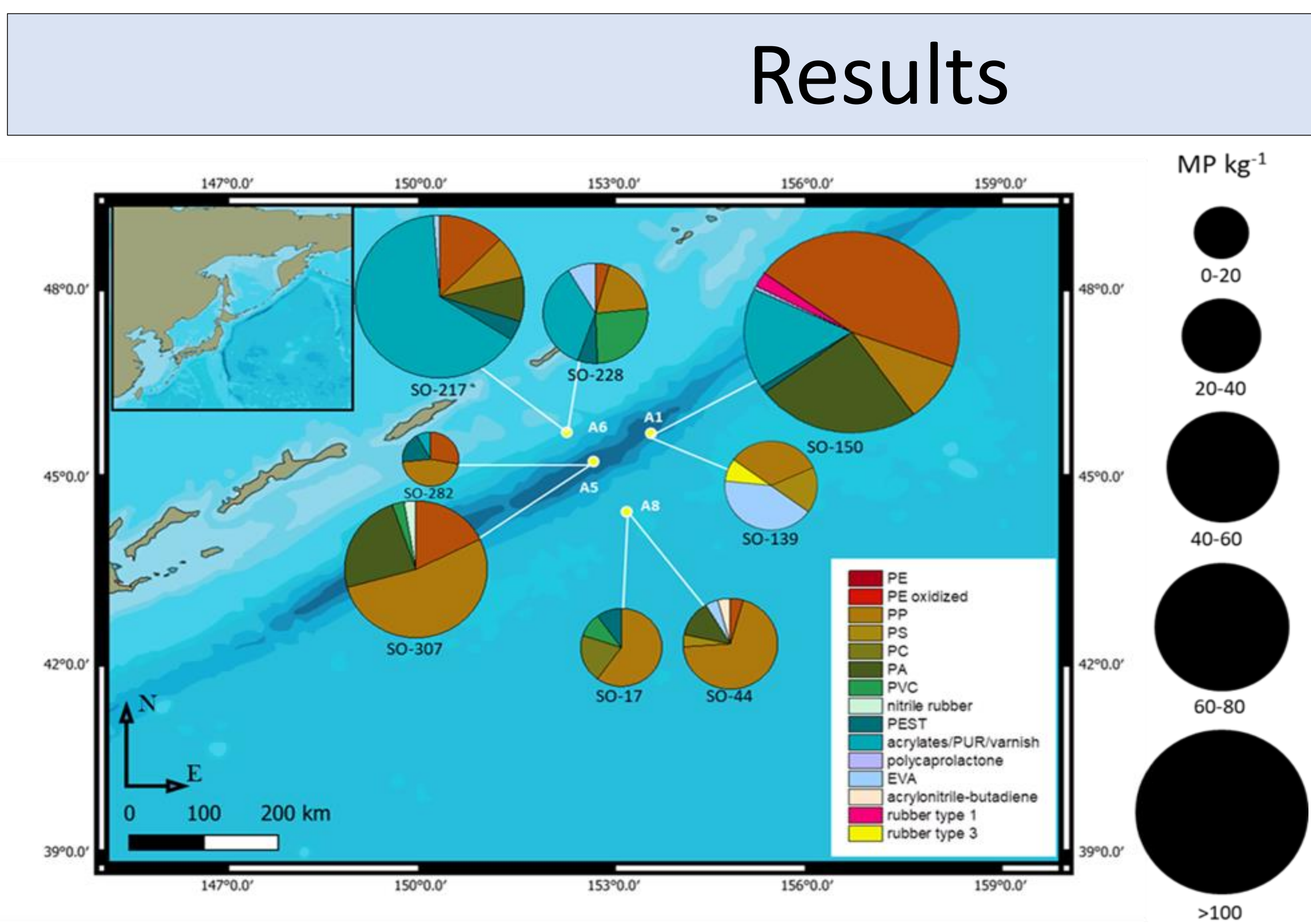

Fig. 1 distribution of microplastic concentration and relative polymer composition in sediments from the Kuril Kamchathe trench. PE: polyethylene, PP: polypropylene, PS: Dolystyrene, PC: polycarbonate, PA: polyamide, PVC: polyvinyl chloride, PEST polyester/polyythylene terephthalate, PUR: polyurethane, EVA: ethylene vinyl acetate.
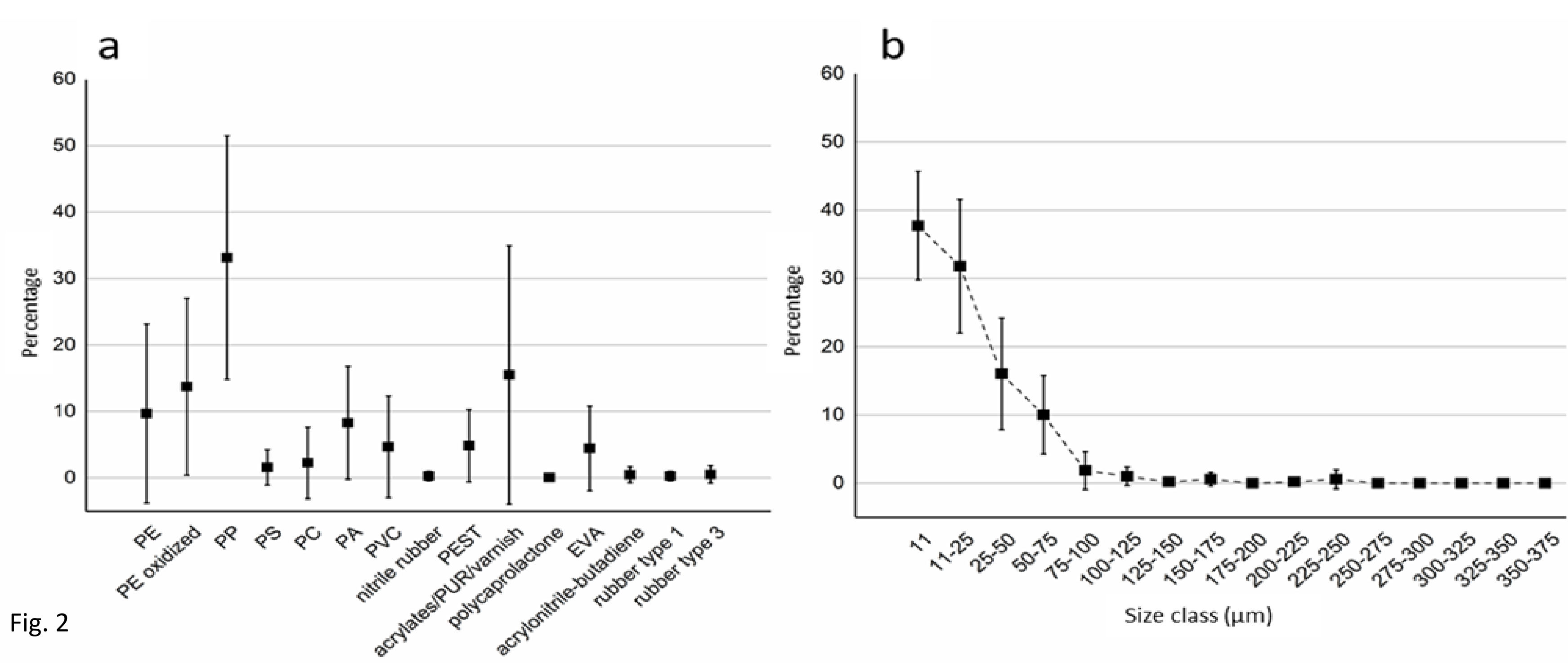

Mean percentage of each polymer type in all sediment samples (black square) PE: polyethylene, PP: polypropylene, PS: polystyrene, PC: polycarbonate, PA: polyamide, PVC: polyvinyl chloride, PEST: polyester/polyethylene terephthalate, PUR: polyurethane, EVA:
ethylene vinyl acetate. Whiskers show the $95 \%$ confidence interval. (b) Mean percentage of each size class in $\mu m$ for all sediment ethylene vinyl acetate. Whiskers show the $95 \%$ confidence inte
samples (squares). Whiskers show the $95 \%$ confidence interval.
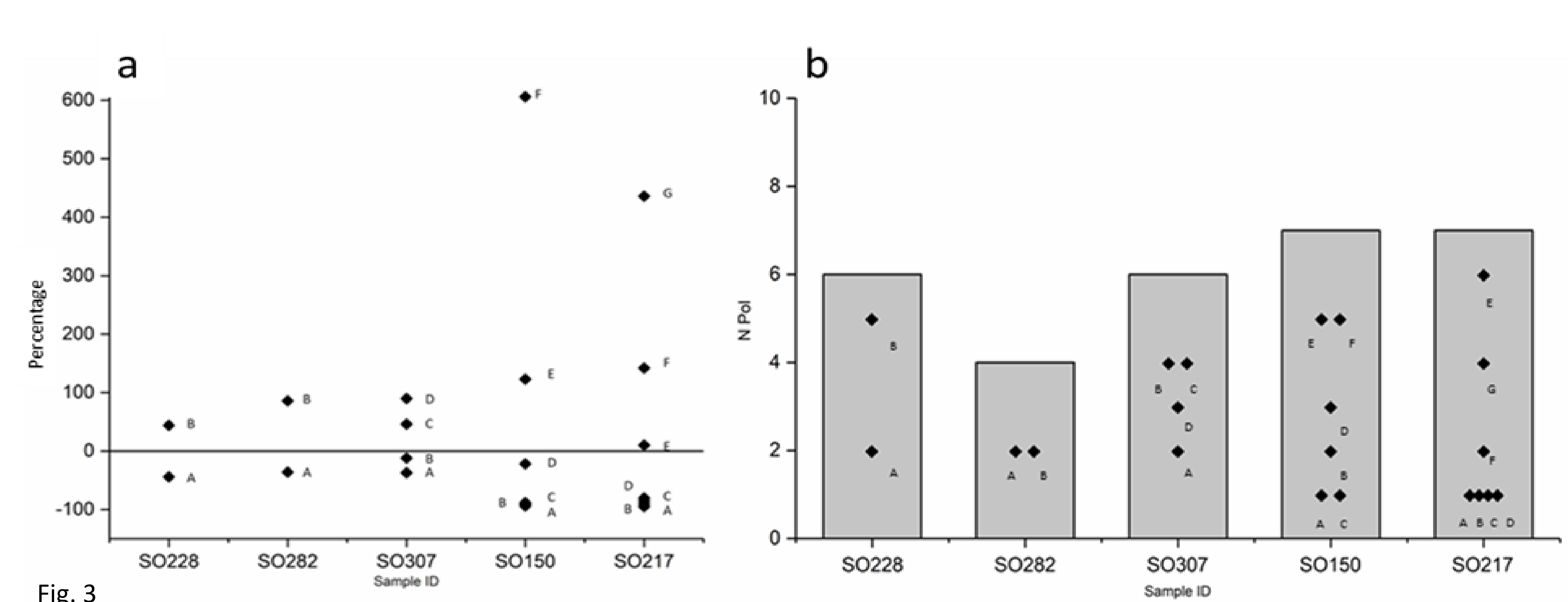

(a) Variation of the estimated Microplastic concentration per kg sediment (dry weight) in the aliquots from the microplastic
concentration in the sample, reported in relative presentence. (b) Polymer diversity in samples. Grey bars depict microplastic polymer in the sample. Symbols depict plastic polymers in aliquot.
MP from eight sediment samples, representing four sampling stations in the abyssal and hadal part of the KKT, were
successfully extracted and analysed. MP $14 \mathrm{~kg}^{-1}(\mathrm{SO} 282)$ to $209 \mathrm{~kg}^{-1}(\mathrm{SO} 150) \mathrm{MP}$ polymer diversity detected ranged from four (SO282) to seven (SO44, SO217 and so150). In Fig. 1 MP concentration per diversity detected in the sampling stations are displayed, with the pie-charts' sizes depicting MP concentration for each corresponding sample site.

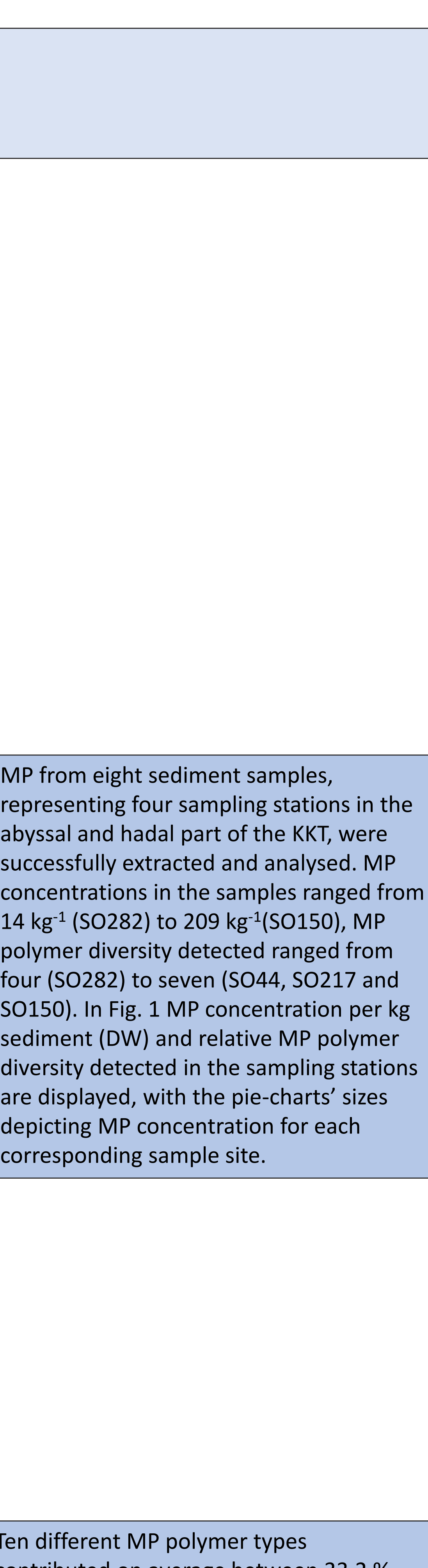
on average between $33.2 \%$ bundance of polymer types over all samples while five polymer types contributed less than $1 \%$ (in descending 3, nitrile rubber, rubber type 3 and polycaprolactone

All MP particles were smaller $375 \mu \mathrm{m}$ (Fig. 2b.) with $99 \%$ smaller than 125
and $71.7 \%$ smaller than $25 \mu \mathrm{m}$ 